\title{
An Approach to Patient with Chorea: A Case Report
}

\author{
Asadi Shiva Prasad*, Thatipelli Ravi Chander, Farmiza Begum, Sandela Anjith Kumar, Nagabelli \\ Nithesh
}

Department of Pharm.D, Vaagdevi Pharmacy College, Government Hospital, Warangal, 506001, Telangana, INDIA.

\begin{abstract}
Chorea is a movement disorder that causes involuntary, unpredictable body movements. It is one of a group of neurological disorders called dyskinesias which is abnormal, uncontrolled, involuntary movement. It can affect one body part, such as an arm, leg or the head, or it can spread over the entire body. Chorea is the rarest disorder and can also effect to one side of body such as chorea of one arm but not both and is referred as hemichorea. Pathophysiology of chorea is somewhat complex and results from dysfunction of network between motor nucleus of thalamus and subcortical nuclei including globus pallidus interna. It is caused genetically or may be acquired. Huntington's disease is most common genetic cause of chorea. Clinical manifestations of hungtintons disease are mainly neurological and psychiatric. Diagnosis of chorea is mainly clinical and family history is important in diagnosis of genetic causes of chorea. Neuroimaging may also help in the diagnosis. Metabolic disorders and some drugs are very important causes of Non-genetic chorea. There is no standard course of treatment for chorea. Treatment depends on the type of chorea and the associated disease. Although there are many drugs that can control it, no cure has yet been identified. Among which includes penicillin prophylaxis and drugs such as sodium valproate and carbamazepine. Here we are reporting a case of 12-year-old female with chief complaints of involuntary movements of hands, feet, which was diagnosed as Chorea based on MRI Scan where it was observed that a ring was formed in the left cerebral hemisphere.
\end{abstract}

Key words: Chorea, Dyskinesias, Involuntary movements, Neuroacanthocytosis, Wilsons disease, Hungtinton's disease like syndrome.

\section{INTRODUCTION}

Chorea is defined as abnormality of movements of limbs and it's a group of neurological disorder called dyskinesia. If only one arm of the body or one side of body is involved is termed as hemichorea. Chorea is presented by semi directed, irregular movements that are not repetitive or rhythmic and appears to follow from one muscle to next. ${ }^{1}$ It can effect body parts such as arms, neck, face, tongue, upper and lower extremes or else it can spread to whole body. ${ }^{2}$ If it is associated with ballismus it is called as choreoballistic movement.

Movement in chorea may be simple or complex and may be superimposed with voluntary actions leading to a bizarre character. Flowing nature of chorea is most characteristic when superimposed with voluntary action. ${ }^{3}$ Hypotonia is a consistent feature and knee jerks may be pendular, motor impersistence is experienced by patients in chorea i.e., they cannot maintain a poster and they squeeze and release the object when ever attempts were made to hold them and poping in and out of tongue can be observed (Harlequin`s tongue) and common attempts are made to mask the symptoms of chorea by voluntary augmenting with semi purposeful movements. ${ }^{4}$ It may be genetical or acquired, genetically may be due to neurodegenerative disease classical Huntington disease or Phenocopy syndrome like Huntington disease like syndrome like type 1, 2, 3 etc., acquired may be due to cerebrovascular disease, streptococcal infections, metabolic disorders, systemic lupus erythromatosus, thyrotoxicosis, coeliac disease $\mathrm{e}^{5}$ and some drugs like levodopa, anti-convulsants, anti-psychotics. Chorea gravidarum refers to the choreic symptoms that occurs during pregnancy. ${ }^{6}$
DOI: 10.5530/ijopp.12.1.14

Address for correspondence: T. Ravi Chander, Associate Professor Head, Department of Pharmacy Practice, Vaagdevi Pharmacy College, Bollikunta, Warangal, 506001, Telangana, INDIA, Phone no: +91 9291565755 Email Id: trc2884@gmail.com

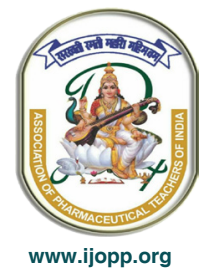




\section{Pathogenesis}

It is complex, generally cortex is responsible for transmitting of impulse for movements of limbs i.e., motor function and prefrontal complex is responsible for the limb movements. Sub thalamus will initiate the motor impulse which activates medial pallidum, which inhibit the cerebral cortex to show their action, in this way they induce parkinsonism. Whereas in chorea or dyskinesia (involuntary muscle movements) the inhibition of impulse from sub-thalamic nucleus increases the motor activity i.e., chorea originates from dysfunction of neural network between motor cortex and basal ganglia which includes sub cortical nuclei Globus Pallidus interna (GPi) and externa (GPe), caudate nuclei, sub thalamus and thalamus. ${ }^{1-7}$ Dysfunction inhibitory GABAergic projections from GPi to motor nucleus of thalamus leads to hyperkinetic choreform movements.

Diagnosis includes patient history, Electroencephalogram (EEG), heamatological investigations, Computed tomography, Magnetic Resonance Imaging (MRI), Electromyography (EMG), is found to be useful in some patients. ${ }^{8-9}$ Treatment depends on the type of chorea and associated disease. Yet no cure has been identified, but there are many drugs that can control it, such as dopaminergic antagonist, ${ }^{10}$ tetrabenazine, ${ }^{11}$ anti-psychotics, ${ }^{12-13}$ anti-convulsants are presently being used. ${ }^{14-15}$

\section{CASE REPORT}

A female patient of age 12 years, was admitted in hospital with chief complaints of involuntary movements of hands, feet and neck since 3 days and she has the previous history of knee pain, headache (occasionally), pharyngitis (2 months back), arthralgia (2 months back), with fever (2 months back). No genetic history with chorea symptoms was found. It was proved that she was suffering with chorea i.e., on MRI Scan- "it was observed that a ring was formed in the left cerebral hemisphere", hence finally diagnosed that she was suffering with chorea. She was prescribed with abnormal, uncontrolled, involuntary movement. It can affect one body part, such as an arm, leg or the head, or it can spread over the entire body. Tab. Haloperidol $(0.01 \mathrm{mg} / \mathrm{kg})$, Tab. Sodium valproate $(10 \mathrm{mg} / \mathrm{kg} /$ day). After 3 days, the dose of both tablets was increased i.e., $0.25 \mathrm{mg}$ and $200 \mathrm{mg}$ of haloperidol and sodium valproate respectively as the symptoms did not reduce. Patient was normal after 5 days (from the day she was admitted). Situs Solitus and Levocardio were recorded and along with the drugs Inj. Penidine 6lakh/i.m Stat, Tab. MVT, ORS were also prescribed.

\section{DISCUSSION}

Primary Motor Cortex or M-1 is one of the principle brain areas involved in the motor function which is located in the frontal lobe of brain with blump called Precentral Gyrus. The role of primary cortex is to generate neural impulses that control the execution of movement from sub thalamus via., medial pallidus they receives signals to inhibit or to exhibit the movement of arms

\section{Subthalamus $\rightarrow$ Medial pallidus $\rightarrow$ Induce Involuntary Movements.}

But in this case, the patient is diagnosed with ring lesions on the left cerebral hemisphere (on the region of cortex). So, the patient is receiving altered signals from the subthalamus (altering the signals) via medial pallidum so, the cortex is showing involuntary movements of the forelimbs, hindlimbs and neck.

Chorea is a neurological disorder. Main cause is Huntington's disease which is also a neurodegenerative disease. Chorea which is caused by huntington's disease is formly called as Huntington's Chorea (most common type).

In Huntington's disease on the short arm of $4^{\text {th }}$ chromosome there is a gene called Huntington (HT'T), which is responsible for the production of proteins called Huntington protein. These have genetic codon which is a nucleotide triplet CAG (Cytosine-Adenine-Guanine), which encodes for glutamate, whenever mutation occurs on this gene more than 36 or above triplet codons are produced which are toxic to the human cells particularly to the brain cells (basal ganglion). ${ }^{16-20}$

Other genetic cause of chorea are rare they include classical Huntington's disease mimic or Phenocopy syndrome called Huntington's Disease Like Syndrome (HDL-syndrome). ${ }^{6,21}$ HDL syndrome occurs in people with characteristic features of Huntington's Disease, who don't have mutations in CAG (nucleotide triplet) present on $4^{\text {th }}$ chromosome. The gene is typically associated with the disorder and other type of chorea is Sydendham's chorea caused as a complication of streptococcal infections. ${ }^{4}$ Chorea gravidum is also a rare type of chorea which presents with involuntary abnormal movement, characterized by abrupt, brief, non-rhythmic, non-repetitive movement of any limb, often associated with non-patterned facial grimaces that occur during the pregnancy. $5,7,22$

Generally, for the treatment of chorea, Dopaminergic antagonists, Antipsychotics, Sodium Channel blockers, Antibiotics were prescribed. A Dopaminergic antagonist 
helps in decreasing the dopamine levels. So that the patient will not be in the excitatory condition. As we know that dopamine helps in the movement of forearms and hindarms, hence by inhibition of these dopamine levels we can manage the involuntary movements in chorea patients. But sometimes this may lead to Parkinsonism because if dopaminergic antagonists are taken regularly they decreases the ability to produce dopamine leading to dopamine deficiency, eventually parkinsonism. So, it is important to maintain the dose of dopamine antagonists. This can also be overcome by prescribing antipsychotics because these drugs are also dopamine antagonists (both $1^{\text {st }}$ and $2^{\text {nd }}$ generation's antipsychotics) which helps in maintaining the levels of dopamine. Some bacterial infections like streptococcal infections also cause chorea hence antibiotics are prescribed to those patients. Measures to reduce the ring lession by anticoagulants (if any clot), neoplastic drugs (if any cancer), Anti-hyperlipidemic Drugs (if any sclerosis), should be prescribed to the patient. ${ }^{10-12}$

\section{CONCLUSION}

In this case as the patient has not undergone with the gene mutations i.e, Huntington's Gene is not mutated. Hence it is Huntington's Disease Like Syndrome (Hdl-S).

In this case directly dopamine antagonist has not been prescribed but antipsychotic drugs were given, which indirectly blocks the dopamine receptors (D-2). Thus, decreasing the dopamine levels. Initially patient did not respond to the treatment (does not decrease symptoms) hence, dose was increased.

\section{ACKNOWLEDGEMENT}

We are grateful for all the respondents who contributed in this study.

\section{CONFLICT OF INTEREST}

The author declared no conflict of interest.

\section{ABBREVIATIONS}

GPi: Globus Pallidus Interna; Gpe: Globus Pallidus Externa; EMG: Electromyography; EEG:
Electroencephalogram; HDL-syndrome: Huntington's Disease like Syndrome; CAG: Cytosine Adenine Guanine; MVT: Multi Vitamin Tablet; ORS: Oral Rehydration Salt.

\section{REFERENCES}

1. Cardoso F, Seppi K, Mair KJ, Wenning GK, Poewe W. Seminar on choreas. Lancet Neurol. 2006;5(7):589-602.

2. Cardoso F. Huntington disease and other choreas. Neurol Clin. 2009;27(3):719-36.

3. Lasker AG, Zee DS. Ocular motor abnormalities in Huntington's disease. Vision Res. 1997;37(3):639-45.

4. Berman SA, Chorea, Joseph AB, Young RR. Movement Disorders in Neurology and Neuropsychiatry. $2^{\text {nd }}$ ed. Malden, Mass: Blackwell Science. 1999;481-94.

5. Gillian B, Sarah T, Lesley J. Huntington's disease, $4^{\text {th }}$ ed. Oxford University Press. 2014.

6. Wild EJ, Tabrizi SJ. The differential diagnosis of chorea. Practical Neurology. 2007;7(6):360-73.

7. Bushara KO. Neurologic presentation of celiac disease. Gastroenterology. 2005;128(4 Suppl 1):S92-7.

8. Bhidayasiri R, Truong DD. Chorea and related disorders. Postgrad Med J. 2004;80(947):527-34.

9. Sanjay P. Chorea-review article. Journal of the Association of Physicians of India. 2013;61:471-83.

10. Pokrovskaia ZA, Insarova NG. The EEG characteristics of patients with Huntington's chorea and their clinically healthy relatives. Zh Nevropatol Psikhiatr Im SS Korsakova. 1988;88(3):22-6.

11. Faustino PC, Terreri MT, DaRocha AD, Zappitelli AC, Lederman HM, Hilario MO. Clinical, laboratory, psychiatric and magnetic resonance findings in patients with Sydenham chorea. Neuroradiology. 2003;45(7):456-62.

12. Bonelli RM, Wenning GK. Pharmacological management of Huntington's disease: an evidence-based review. Curr Pharm Des. 2006;12(2):701-20.

13. Huntington Study Group. Tetrabenazine as antichorea therapy in Huntington disease: a randomized controlled trial. Neurology. 2006;66(3):66-72.

14. Bonelli RM, Hofmann P. A systematic review of the treatment studies in Huntington's disease since 1990. Expert Opin Pharmacother. 2007;8(2):141-53.

15. Patterson JF. Treatment of Chorea Gravidarum with Haloperidol. Southern Medical Journal. 1979;72(9):1220-1.

16. Mestre T, Ferreira J, Coelho MM, Rosa M, Sampaio C. Therapeutic interventions for symptomatic treatment in Huntington's disease. Cochrane Database Syst Rev. 2009;8(3):456.

17. Phillips W, Shannon KM, Barker RA. The current clinical management of Huntington's disease. Mov Disord. 2008;23(11):1491-504.

18. Raymund ACR. Hungtinton's disease- A Clinical Review. Orphanet Journal of Rare Diseases. 2010;5(1):40.

19. Huntington's disease collaborative research group. A novel gene containing a trinucleotide repeat that is expanded and unstable on Huntington's disease chromosomes. Cell. 1993;72(6):971-83.

20. Wheelock VL, Tempkin T, Marder K, Nance M, Myers RH, Zhao H, et al. Predictors of nursing home placement in Huntington disease. Neurology. 2003;60(6):998-1001.

21. Aziz NA, Burg JMVD,, Landwehrmeyer GB, Brundin P, Stijnen T, Roos RA, et al. Weight loss in Huntington disease increases with higher CAG repeat number. Neurology. 2008;71(19):1506-13.

22. Trottier $\mathrm{Y}$, Biancalana V, Mandel JL. Instability of CAG repeats in Huntington's disease: relation to parental transmission and age of onset. J Med Genet. 1994:31(5):377-82. 